cutent aujourd'hui sous nos yeux, déchaùnent les avalanches, engendrent des torrents, des laves et rendent toujour's plus irrég"ulier le débit de nos fleuves, de nos rivières et ont, par suite, sur le r'égime du Rihône, la plus désastreuse influence.

L'Eitalt ne saurait donc, à lui seul, suffire à la tâche énorme de restaurer toutes les montagnes. Si, seul, il peut et doit cicatriser les plaiest les plus dangereuses qui menacent de tout engloutir, c'esit aux particulier's et surtout aux communes propriétaires de tant de friches qu'il appartient de créer les vastes massifs forestier's qui seront à la fois la richesse et la sauvegin"de du pays tout entier.

\section{Les Installations Hydro-Électriques de la Société Énergie Électrique du Littoral Méditerranéen}

La France possède, à l'heure actuelle, d'importants réseaux de distribution d'énergie électrique alimentés par la houille blanche. Parmi ces réseaux, nous citerons celui de la Société Grenobluise de Force et Lumière, dont les fils couvrent le département de l'Isère, à la manière d'une vaste toile d'araignée s'étendant jusque dans les départements du Rhône et de la Loire. Nous citerons encore le réseau de la Sociélé méridionale de Transport de force, qui s'étend.sur tout le département de l'Aude. Mais le plus développé de tous ces réseaux est bien certainement celui de la Société Energie Electrique du Littoral Méditerranéen; dont le distingué directeur général est M. C. Cordier, ingénieur' en chef des Ponts et Chaussées, président du Syndicat des. Forces hydrauliques, réseau qui s'étend, à l'heure actuelle, sur plus de quatre départements, le long de la Méditerranée. La houille blanche sur la Côte d'azur !

Le réseau hydro-électrique de la Société Énergie Électrique comprend tout le bassin du Var, ainsi que celui de la Durance, et utilise les eaux qui tombent sur le vaste espace compris entre la frontière italienne, le massif du Pelvoux, le Vercors, le Rhône et la mer. Bien que cette région soit fort déboisée, el subisse les funestes effets dûs à la transhumance, le régime auquel sont soumises les usines de la Société n'est pas trop défavorable. En effet, d'une part, plusieurs rivières prennent leur source dans la haute montagne et possèdent, sous forme de neige ou de glace, des réserves de houille blanche qui, accumulées durant l'hiver, régularisent le débit pendant l'été. D'autre part, l'ensemble du bassin hydrologique est à une altitude modérée, et dans le voisinage immédiat de cette vaste chaudière à évaporation intense qu'est la Méditerranée, de sorte que les précipitations atmosphériques s'y font abondamment en hiver sous forme de pluie, ce qui rend l'étiage d'hiver relativement abondant par rapport aux cours d'eau à régime purement glaciaire du haut Dauphiné et des Savoies. Certaines rivières, comme l'Argens ou la Siagne, ont leur bassin établi tout entier à basse altitude. Dans son ensemble, le régime hydraulique auquel est soumis l'Énergie Électrique est relativement régulier, avec maximum en hiver, ce qui est tout à l'avantage de cette Société, la période de grande consommation ayant précisément lieu pendant la saison froide, surtout pour toute cette région maritime qui s'étend depuis 'Toulon jusqu'à la frontière italienne, et qui constitue la Côte d'azur $\left(^{\star}\right)$.

(") Pour une partie de cette étude, nous avons eu recours à la conférence faite sur ce sujet, à la Société des Ingénieurs civils de France, par M. DE MARCHENA (voir. Bulletin de juillet 1907), ainsi qu'aux articles sur le" même sujet publiés dans l'Eclairage Electrique sous la signature de M. FrRILLY.
Comme on va le voir par la suite, les installations de l'Énergie Electrique sont un remarquable exemple de l'emploi judicieux de stations centrales à vapeur de secours, placées dans le voisinage immédiat des lieux de consommation.

En outre de l'Énergie Electrique, nous dirons aussi quelques mots d'une de ses filiales, le sud Electrique.

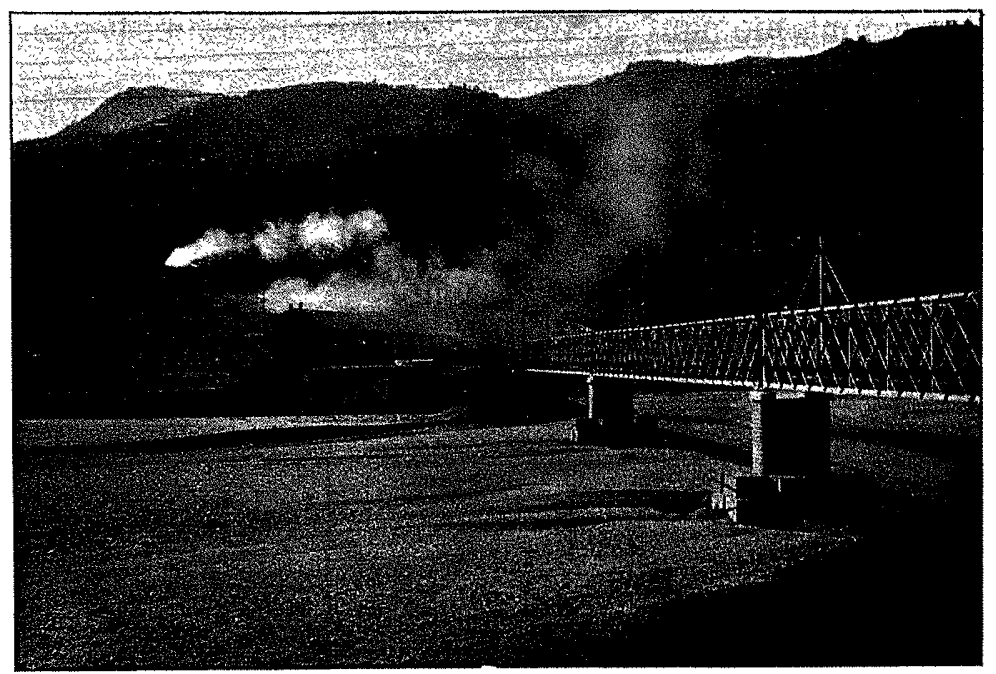

FıG. 1. - Vue de lusine génératrice du plan-rlu-Var el de la fabrique de Carbure de Galcium de la Société Niçoise d'Electrochimic

Historique. - En 1898-1899, la Compagnie des Tramways de Nice et du Littoral, filiale de la Compagnie française Thomson-Houston, coupait, au moyen d'un canal souterrain, la boucle que le Var forme à «la Mescla», aussitôt après son confluent avec la Tinée, et, au moyen d'une dérivation de 600 mètres créait, à 30 kilomètres de Nice, une chute de 10 mètres de hauteur, d'une puissance de 2000 chevaux environ. A la même époque, la Compagnie des Grands Travaux de Marseille étudiait un projet grandiose, celui de l'application de l'électricité à la traction des trains entre Cannes et Vintimille, et s'assurait les droils de riveraineté pour la création d'une chute de $3000 \mathrm{HP}$ sur la rivière le Loup. Le transport d'énergie de la Mescla ayant donné d'excellents résultats, les deux grandes Sociétés précitées décidèrent, en juin 1900, d'associer leurs efforts pour utiliser les ressources hydrauliques de la Provence, au moyen de transports d'énergie à grandes distances, et en faire bénéficier, non seulement les localités voisines, mais encore celles de la côte méditerranéenne, et, à cet effet, elles fon-

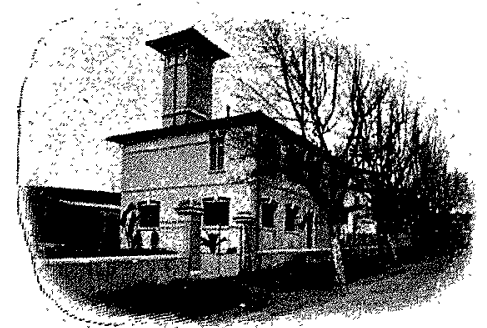

Fig. 2. - Sous station dAntibes. dèrent la Socièté Énergie Électrique du Littoral Mé. diterranéen.

Le programme que cette Sociélé s'est appliquée à réaliser est le suivant :

10 Transporter et distribuer l'énergie électrique, produite par des usines hy dro-électriques, dans toute la région comprise entro le Rhône, le Dauphiné, les Alpes et la Méditerranée

2o Relier entre elles les usines hydro-électriques établies sur des cours d'eau de régimes différents, de manière à équilibrer ces régimes, et à diminuer l’influence des éliages qui se produisent généralement en hiver sur les rivières d'origine glacière (Var, Durance), et en été sur les autres rivières (Loup, Siagne, Verdon, etc.).

$3^{\circ}$ Disposer ses réseaux de manière à assurer une grande sécurité d'exploitation, et à permettre de répartir', suivant les besoins, la charge entre les diverses usines genéra- 
trices, sans être obligé de les faire toutes marcher en parallèle à grande distance, ce qui n'est pas sans présenter de grosses difficultés et de sérieux inconvénients;

$4^{\circ}$ S'assurer le plus grand nombre possible d'usines à vapeur, destinées tant à assurer le service de secours, en cas d'interruption des lignes, qu'à permettre de faire face àux besoins de la clientèle en cas de pénurie d'eau extraordinaire ;

$5^{\circ}$ Disposer de chutes d'une puissance suffisante pour être à tout moment en mesure de faire face aux besoins des régions desservies;

$6^{\circ}$ Se spécialiser dans la vente en gros, en laissant le

\section{RÉSEAU DES ALPES-MARITIMES}

Le réseau des Alpes-Maritimes est alimenté normalement par les trois usines hydrauliques précitées, de la Mescla, du Plan-du Var et du Loup. Mais, pendant la période d'hiver, ce réseau a à faire face à des besoins très supérieurs à ceux de la moyenne de l'année, et, en outre, dans une même journée, ces besoins atteignent un maximum très accusé aux heures d'éclairage. Aussi, pour y faire face dans des conditions rationnelles, l'Énergie Électrique dut avoir recours à cinq usines à vapeur : celles de Sainte-Agathe et du Risso, à Nice; de Mougins, de Menton et de Monaco.

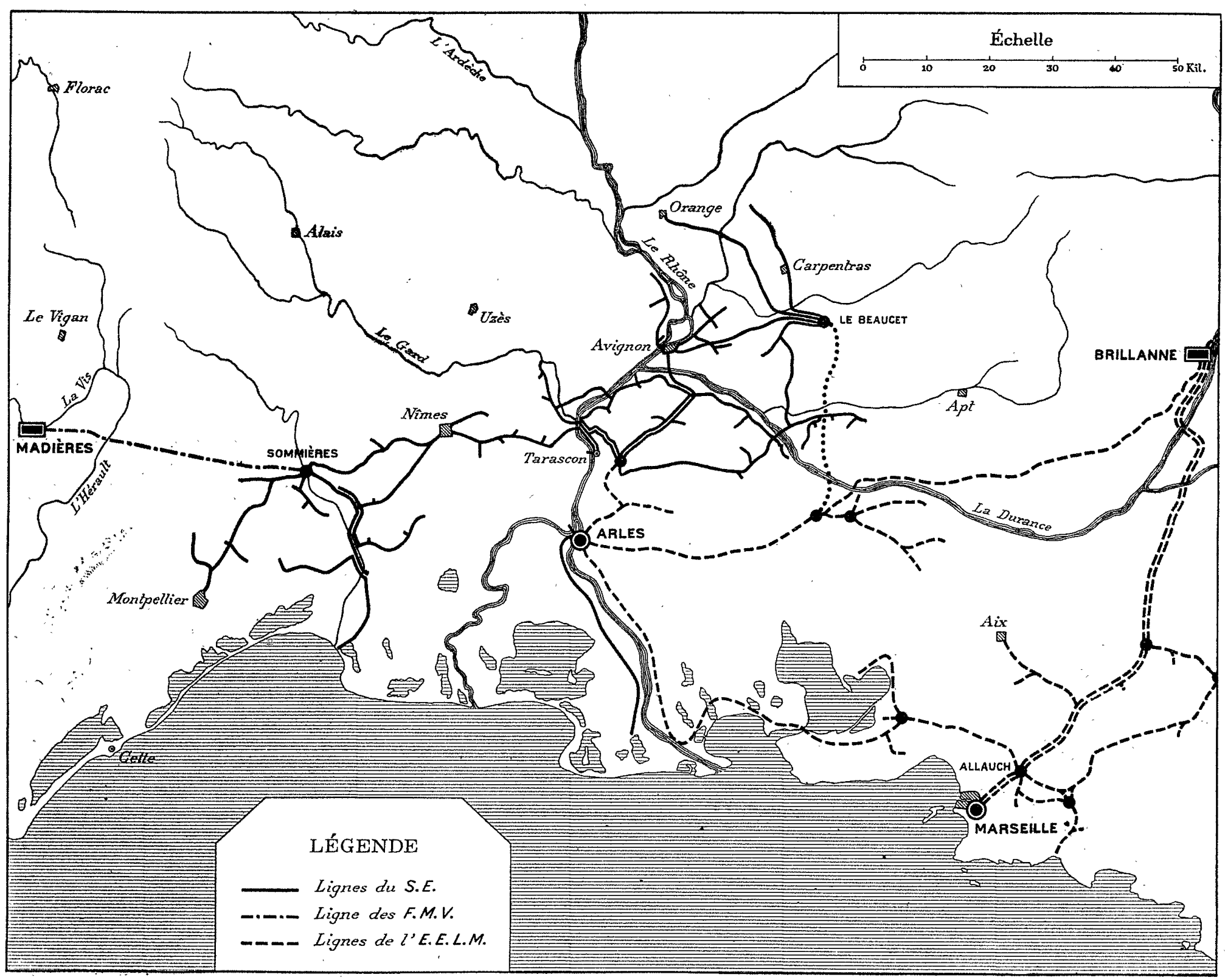

RÉSEAU DU SUD-ÉLEGTRIQUE ET LIGNeS QUI L'ALIMENTENT

plus possible à des clients ou à des Sociétés filiales le soin de faire la distribution de détail.

L'Énergie Électrique créa tout d'abord l'usine de Pataras, sur le Loup (3000 HP), pendant qu'elle rachetait à la Cie des Tramways de Nice son usine de la Mescla, ainsi que la ligne à 10000 volts qui reliait cette usine aux tramways de Nice. Elle rachetait bientôt également à la Société des Forces motrices des Alpes-Maritimes son usine du Plandu-Var (5000 HP); aussi, dès 1902, l'Énergie Électrique disposait de plus de $10000 \mathrm{HP}$, dont 3000 étaient affectés au service de l'usine de carbure de calcium de la Société Nicoise d'Electrochimie, établie à côté de l'usine du Plan-du-Var.
L'usine de la Mescla utilise une chute de 10 mètres de hauteur, avec un débit variant de 15 à 20 mètres cubes. Elle comprend trois turbines Escher-Wyss de $750 \mathrm{HP}$, directement accouplées chacune à un alternateur produisant du courant triphasé à 10000 volts, 25 périodes. De cette usine partent deux lignes la reliant, l'une à l'usine du Plan-du-Var, l'autre à Nice.

Le cánal de dérivation de l'usine du Plan-du-Var, ainsi d'ailleurs que celui de l'usine de la Mascla, est.prévu pour pouvoir débiter $20 \mathrm{~m}^{3}$ à la seconde. Comme la chute est de 25 mètres, la puissance produite varie de 3700 à 5000 che. vaux, sur lesquels $3000 \mathrm{HP}$ sont constamment réservés à la 
fabrique de carbure de calcium dé la Société niçoise d'Electrochimie dont l'usine est accolée à l'usine hydro-électrique, le surplus étant affecté au réseau général de l'Énergie Électrique et dirigé sur Nice.

Pour ce qui regarde ce dernier service, l'usine du Plandu-Var comporte 3 turbines doubles de 900 chevaux, à axe horizontal et du système Francis, tournant à 375 tours, et directement accouplées chacune à un alternateur produisant du courant triphasé à 10000 volts, 25 périodes. Les turbines fonctionnent sous une aspiration de $6^{\mathrm{m}} 75$.

L'usine de Pataras, sur le Loup, utilise une chute de 250 mètres, avec un débit cui tombe rarement au-dessous surtout sa contribution aux heures les plus chargées pour passer les pointes d'éclairage, grâce à sa haute chute et à son réservoir régulateur.

L'usine du boulevard Ste-Agathe est plus spécialement affectée au service des tramway de Nice et du Littoral (*). Elle fonctionne tantôt comme sous-station de transformation de courant, et tantôt comme usine à vapeur de secours. A cet effet, elle comprend 2 moteurs synchrones de $700 \mathrm{Kw}$. sous 10000 volts, qui actionnent chacun une dynamo à courant continu, 550 volts, mais qui peuvent être aussi entraînés au moyen de courroies par une machine à vapeur; dans ce dernier cas, le moteur synchrone fonc-

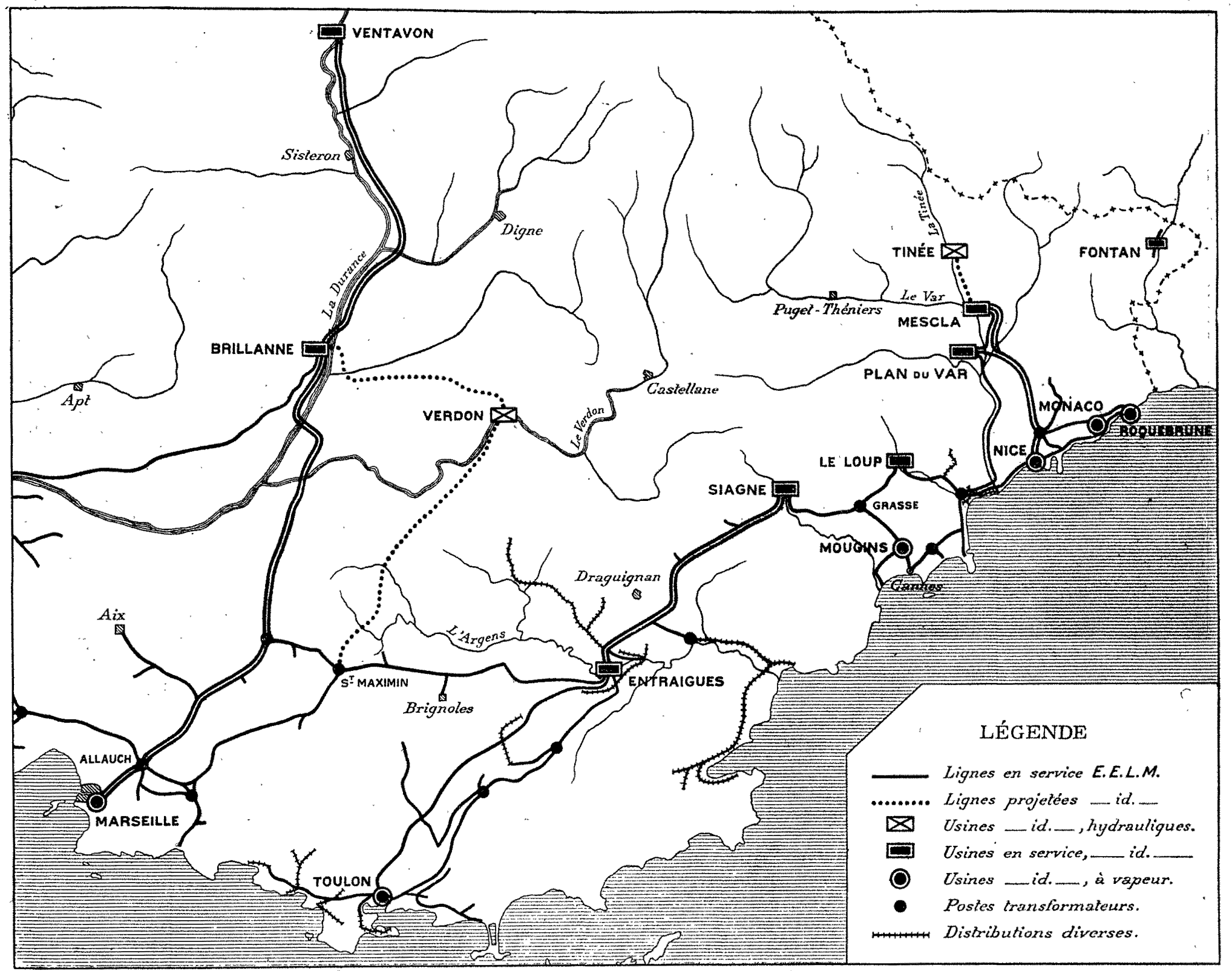

HÉSEAU DE L'ÉNERgIE ÉLECTRIQUe DU LITTORAL MÉDITERRANÉEN

d'un mètre cube par seconde. Le canal de dérivation a été établi pour le débit de $1 \mathrm{~m}^{3}$, et se termine par un réservoir compensateur de $6500 \mathrm{~m}^{3}$, permettant d'augmenter considérablement la puissance instantanée de l'usine au moment du passage des pointes. Il y a quatre turbines Escher-Wyss de $1000 \mathrm{HP}$, directement accouplées à un alternateur débitant également du courant triphasé à 10000 volts, 25 périodes. De cette usine partent deux lignes se dirigeant, l'une vers Nice, l'autre vers Grasse.

Les usines du Var et celle du Loup se complètent mutuellement, celles du Var effectuant toute la partie constante de la consommation journalière, et celle du Loup apportant tionne comme alternateur et renvoie du courant sur le réseau. L'usine comprend aussi 2 groupes moteurs-générateurs de 250 kilowatts.

L'usine à vapeur du boulevard Risso comporte 3 groupes turbo-alternateurs, à axe vertical, système Curtis, tournant à 1500 tour's par minute, et d'une puissance nominale et 1500 HP. Chaque turbine est à quatre étages de pression, chaque étage comportant une série de tuyères de détente, deux jeux d'aubes mobiles actionnant une même roue, et

(") Voir la Houille Blanche d'octobre 1906, page 233 et suivantes. 
un jeu d'aubes fixes placé entre les aubes mobilès. Les alternateurs sont bipolaires, et produisent du courant triphasé à 10000 volts. La vapeur est fournie par 7 chaudières multitubulaires Babcock et Wilcox produisant 4000 $\mathrm{kg}$. de vapeur à l'heure. Ces chaudières sont munies de surchauffeurs de vapeur, et brûlent du poussier de coke sur des grilles soufflées d'une manière spéciale et refroidies par une injection d'eau pulvérisée. Le coût de premier élablissement de liksine est revenu à 500 francs le kilowatt.

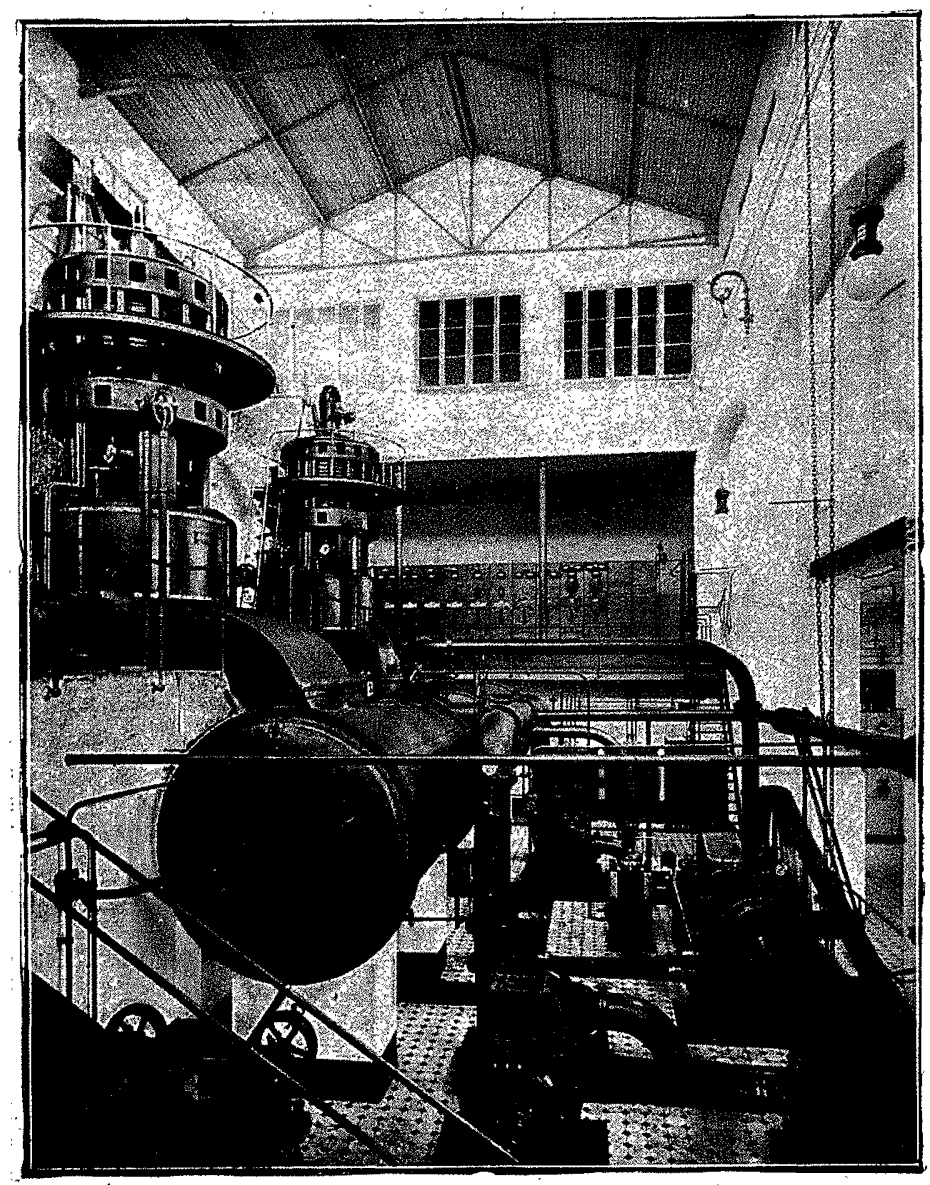

Fia. 3. - Vue des lurbines à vapeur de l'usine du Risso.

L'usine de Mougins, près de Cannes, possède une machine à vapeur, ainsi qu'une petite turbine installée sur le canal d'alimentation de la ville de Cannes. Elle alimente la région Cannes-Grasse, concuremment avec l'usine du Loup et avec celle de la Siagne, dont nous parlerons plus loin.

L'usine de Monaco est alimentée normalement par les usines hydro-électriques établies sur le Var, mais elle peut servir aussi d'usine à vapeur de secours. Elle comporte 3 machines à vapeur horizontales de $150 \mathrm{HP}$, et une de 300 HP; 2 groupes moteurs-générateurs, à 10000 volts, composés d'un moteur synchrone actionnant une dynamo de $200 \mathrm{kw}$, alimentant un circuit d'éclairage à 3 fils, 240 volts; 2 groupes moteurs générateurs de $75 \mathrm{kw}$, alimentés sous 440 volts, et servant d'égalisateurs du circuit précédent; un groupe moteur-générateur de $200 \mathrm{kw}$ à 10000 volts, alimentant un circuit d'éclairage à 120 volts; 2 commutatrices de $150 \mathrm{kw}$, chacune; une batterie d'éclairage de 1250 ampèresheures; une batterie de traction de 460 ampères-heurès; un survolteur de batterie, formé de 2 dynamos à 2 collecteurs, de $24 \mathrm{kw}$, commandées par un moteur asynchrone à 2200 volts.

L'usine de Menton comprend une machine à vapeur de $250 \mathrm{HP} ; 2$ commutatrices de $150 \mathrm{kw}$; une batterie d'accumulateurs de 320 ampères-heures. Elle alimente la région de Menton:
Les installations précédentes ont permis à l'Energie Electrique de desservir la clientèle suivante, acquise assez rapidement: Tramways de Nice et du Littoral; Cie du Gaz et de l'Electricité de Nice; $\mathrm{C}^{\mathrm{i} \theta}$ de distribution d'eau, de lumière et de force, de Monte-Carlo supérieur et extensions; $\mathrm{C}^{\text {ie }}$ d'Electricité du Sud-Est; Cie des Tramways de Cannes ; Chemin de fer à crémaillère de la Turbie; Cie d'éclairage de Villefranche-sur-Mer, Beaulieu et extensions; Cis d'Eclairage électrique de Cannes ; $\mathrm{C}^{\text {ic }}$ des Grands Travaux de Marseille (exploitation de Grasse); exploitation monégasque d'Electricité, etc.

En même temps, la Société prenait et assurait directement la concession de distributions électriques dans un grand nombre de communes: Antibes, Cabbé-Roquebrune (Cap Martin), Vallauris et Golfe Juan, Bar-sur-Loup, Villeneuve-Loubet, Châteauneuf, Gourdon, Saint.Vallier, SaintCézaire, Mouans-Sartoux, Mougins, Mandelieu, La TrinitéVictor, Drap, Contes, Levens, Saint-Martin-du-Var, La Roquelte, Colomars, etc.

Elle traila d'autre part avec le département des AlpesMaritimes pour lui fournir toute l'énergie électrique nécessaire pour l'exploitation du vaste réseau de tramways dont il a entrepris la construction, et qu'il a rétrocédé â la Compagnie des chemins de fer du Sud de la France et à la Compagnie des. Tramways de Nice et du Liltoral.

Le réseau primaire des Alpes-Maritimes est à la tension de 10000 volts, il est bouclé en tous ses points par des réseaux secondaires à 2200 volts, et par des réseaux tertiaires à 230 et 115 volts:

A Nice, dans l'usine de Risso, où convergent les lignes venant des usines établies sur le Var et sur le Loup, et d'où partent celles qui alimentent la région Beaulieu-Monaco, et celles qui désservent les sous-stations des Tramways et du Gaz, on a établi un poste de sectionnement très complet, muni de jeu d'interrupteurs à deux directions, grâce auquel chaque feeder sortant du poste peut être branché sur une quelconque des usines.

Malgréll'étendue considérable du réseau par rapport à la tension choisie, on a pu obtenir en tous les points une régularité de voltage très satisfaisante, grâce à l'emploi de régulateurs aulomatiques de potentiel installés dans les usines hydrauliques, ainsi qu'aux stations de VilleneuveLoubet, à l'extrémité de la ligne du Loup, et de St-Pons, à l'extrémité des lignes venant des usines établies sur le Var.

\section{RÉSEAU DU VAR}

Le réseau du Var est alimenté par les usines hydrauliques d'Entraygues, sur l'Argens, et de St-Césaire, sur la Siagne, et par deux usines à vapeur situées à Toulon. L'usine d'Entraygues ayant été décrite dans La Houille Blanche de janvier 1906, nous y renverrons le lecteur, et nous passerons de suite à l'usine de la Siagne.

Usine de la Siagne. - Cette usine est établie sur le cours supérieur de la Siagne, près-de St-Césaire, sur les confins des départements du Var et des Alpes-Maritimes. Grâce à la pente de la rivière, une dérivation de $7,5 \mathrm{~km}$. a suffi pour créer une chute de $350 \mathrm{~m}$. Le débit minimum de la rivière oscille entre ${ }_{2}^{1}, 5$ et $2,5 \mathrm{~m}^{3}$. Grâce à la création d'un vasté réservoir de $11000 \mathrm{~m}^{3}$, auquel aboutit le canal d'amenée, prévu pour 2400 litres, on a pu aménager l'usine pour un débit de $3 \mathrm{~m}^{3}$, correspondant à une puissance instantanée de 10000 chevaux. L'usine est établie immédiatement à l'amont de la prise d'eau du canal qui alimente la ville dé Cannes, et, comme les besoins d'eau de l'usine et de la ville de Cannes ne sont pas concomittants, on a du créer à l'aval de l'usine un réservoir compensateur d'une capacité égale à celle du précédent, soit de 11000 mètres cubes.

La prise d'eau s'effectue au moyen d'un barrage fixe, un peu oblique au courant, formé d'ún mur en maçonnerie dè 
$21 \mathrm{~m}$. de longueur, dont $12^{\mathrm{m}} 50$, à l'emplacement du lit primitif de la rivière, sont arasés en déversoir. A l'une de ses extrémités se trouve un pertuis de chasse. L'eau, déviée par le barrage, pénètre dans une chambre d'eau, traverse une grille, puis pénètre dans le canal d'amenée en traversant une vanne de réglage. A $200 \mathrm{~m}$. de la prise d'eau se trouve une chambre de décantation, munie d'une vanne de purge, d'un déversoir règlant le plan d'eau; et d'une grille commandant l'entrée de la seconde partie du canal d'amenée. Une dérivation permet de relier directement les deux parties du canal d'amenée, sans passer par la chambre de décantation.

Le canal d'amenée, couvert sur tout son parcours, a une longueur totale de $7500 \mathrm{~m}$., une pente de 2,3 millimètres par mètre, et une section rectangulaire de $1 \mathrm{~m} 10$ de large sur $1 \mathrm{~m} 25$ de haut. Il est établi à flanc de coteau, d'abord sur $2200 \mathrm{~m}$. dans un terrain graveleux ayant necessité la construction d'un dallage continu en béton armé de $50 \mathrm{~cm}$. d'épaisseur, puis sur $3300 \mathrm{~m}$. dans un terrain de calcaire compact; en tranchée sur $300 \mathrm{~m}$. et en souterrains partiellement revêtus sur $1900 \mathrm{~m}$. Un ravin de $60 \mathrm{~m}$. de profondeur a été franchi au moyen d'un siphon en tôle d'acier, de 1 m25 de diamètre et de 4 à $6 \mathrm{~mm}$. d'épaisseur.

moitiè de la vitesse du jet, dépasse $40 \mathrm{~m}$. à la seconde. La vitesse angulaire de rolation est de 375 tours. par minute pour les grosses unités, et de 900 tours pour les petites. Le rendement a, parait-il, atteint 85 pour 10 à pleine charge: Les régulateurs sont à servo-moteur actionné par l'eau de la chute, et sont combinés avec des déchargeurs à fermeture progressive, de manière à éviter les coups de bélier. Au moyen d'un petit moteur électrique de un cinquième de cheval, on peut, du tableau même de distribution. faire varier la vitesse de rotation des turbines

Les alternateurs sont du type à induit fixe et à inducteur tournant; ils sont directement accouplés aux turbines par l'intermédiaire d'un volant en fonte de 4 tonnes, et sont bobinés pour la tension de 11000 volts, 25 périodes; de manière à pouvoir alimenter directement le réseau des Alpes-Maritimes. Ils ont été fournis par la Cie ThomsonHouston, ainsi, d'ailleurs, que tout le matériel électrique employé par l'Energie L'lectrique.

Un régulateur de potentiel, système Thury, permel d'obtenir automatiquement une tension constante sur les barres omnibus.

L'usine de la Siagne alimente à la fois le réseau des Alpes-Maritimes et celui du Var.

Ce dernier est alimenté à la tension de 30000 volls, parl'intermédiaire d'un poste de 9 transformateurs monophasés, à bain d'huile, de $750 \mathrm{kw}$ chacun, groupés par 3 en triangle du côté 11000 volts, et par 3 en étoile du côté 30000 volts.

Le courant qui circule dans le tableau de distribution est ramené à la tension de 110 volts, de maniêre á éviter tout accident. Chaque ampèremètre est branchó sur le secondaire d'un transfor. mateur à air, chaque voltmètro sur le secondaire d'un transformateur à huile. Les interrupteurs haute tension sont du type $\mathrm{H}$ de la Compagnie Thomson-Houston dont on trouvera plus loin la description à propos du poste d'Allauch.

Réseau du Var. - Le réseau du Var, postérieur à celui des Alpes-Marilimes, marque sur celui-ci un réel pl'ogrès au poirt

Le canal d'amenée aboutit au réservoir de $11000 \mathrm{~m}^{3}$, d'où partent deux conduites forcées de $893 \mathrm{~m}$. de longueur. Leur diamètre est de $0 \mathrm{~m} 92$ sur les 430 premiers mètres, et de $0^{\mathrm{m}} 83$ sur les 463 derniers mètres. L'épaisseur des tôles varie de 4 à $22 \mathrm{~mm}$. Les tuyaux sont à double et triple rivure longi. tudinale, et simple rivure transversale. Les conduites sont commandées par deux vannes d'arrêt, et elles partent d'un puisard placé vers le milieu du mur du réservoir (la hauteur de ce mur est de $6^{\mathrm{m}} 60$ du côté de la rivière). Ce puisard a son radier à $50 \mathrm{~cm}$. en contrebas de la génératrice inférieure des conduites, et il est entouré d'une grille, de manière à arrêter les corps flottants.

L'usine de la siagne comprend 4 groupes électrogènes de 2500 HP chacun, avec place réservée pour un cinquième groupe, et 2 groupes de $150 \mathrm{HP}$ pour l'excitations des alternateurs et les services auxiliaires.

Les turbines sont du type Pelton, à axe horizontal, et sortent des ateliers Escher Wyss. Le distributeur est du type à aiguille, et permet de faire varier le débit sans dévier l'axe du jet. Les aubes sont rapportées, en acier fondu, et polies à la meule. Lcur vitesse périphérique, qui est égale à la de vue de l'élevation de la tension qui a été triplée; celte tension y est en effet de 30000 volts.

Une ligne, double entre Sl-Césaire et Entraigues, relie l'usine de la Siagne au poste de St-Maximin, ou se fait, par l'intermédiaire do transformateur's, la jonction avec le réseau des Buuches-du-Rhône, qui marche à 50000 volts. Un poste de sectionnement à Entraigues permet d'alimenter les deux lignes de Toulon par la Siagne, concuremment avec l'usine d'Entraigues. La jonction du réseau du Var avec celui des Alpes-Maritimes se fait à l'usine de la Siagne, qui sert de véritable régulateur de régime.

Des réseaux secondaires à 10000 et 3250 volts facilitent l'alimentation des réseaux tertiaires de distribution à 115 et 230 volts.

Les usines à vapeur de Toulon servent à la fois de sousstalion de transformation du courant et aussi de secours au moment du passage des pointes. Ce sont : l'usine du Brunet (ancienne usine des Tramways) et celle de la Lou. bière (usine de la Société d'éclairage de Toulon). En outre des groupes électrogènes à vapeur, cette dernière usine comporte un groupe à moteur à gaz de 350 chevaux. 
Le transport du Var a été mis en service vers la fin de l'année 1904. Les deux clients principaux qu'il dessert sont la Compagnie des Tramways de Toulon et environs, et la Société d'Eclairage de Toulon, à laquelle il fournit l'énergie dont elle a besoin pour la distribution de l'électricilé, tant dans la commune de Toulon que dans diverses communes voisines (La Garde, La Valette, Le Pradet, Carqueiranne, etc.).

Ce transport alimente en outre la Compagnie Provençale de Tramways Electriques. Il fournit du courant à la Compagnie d'Electricité du Sud.Est pour l'éclairage des communes d'Ollioules, de Reynier, de Sanary, et de la Crau d'Hyères, de Bandol, Cassis. le Beausset, la Cadière, etc. ; à la Société d'Electricité de la Seyne-sur-Mer pour l'éclairage de la Seyne et de Saint-Mandrier ; à la Société de la Nartuby pour l'éclairage des communes du Muy, de Fréjus, de Saint-Raphaël, de Cogolin, de Saint-Tropez, etc.; à la Société électrique de l'Argens pour la distribution de l'électricité dans les communes de Lorgues, Salernes, Flayosc, Aups, etc.

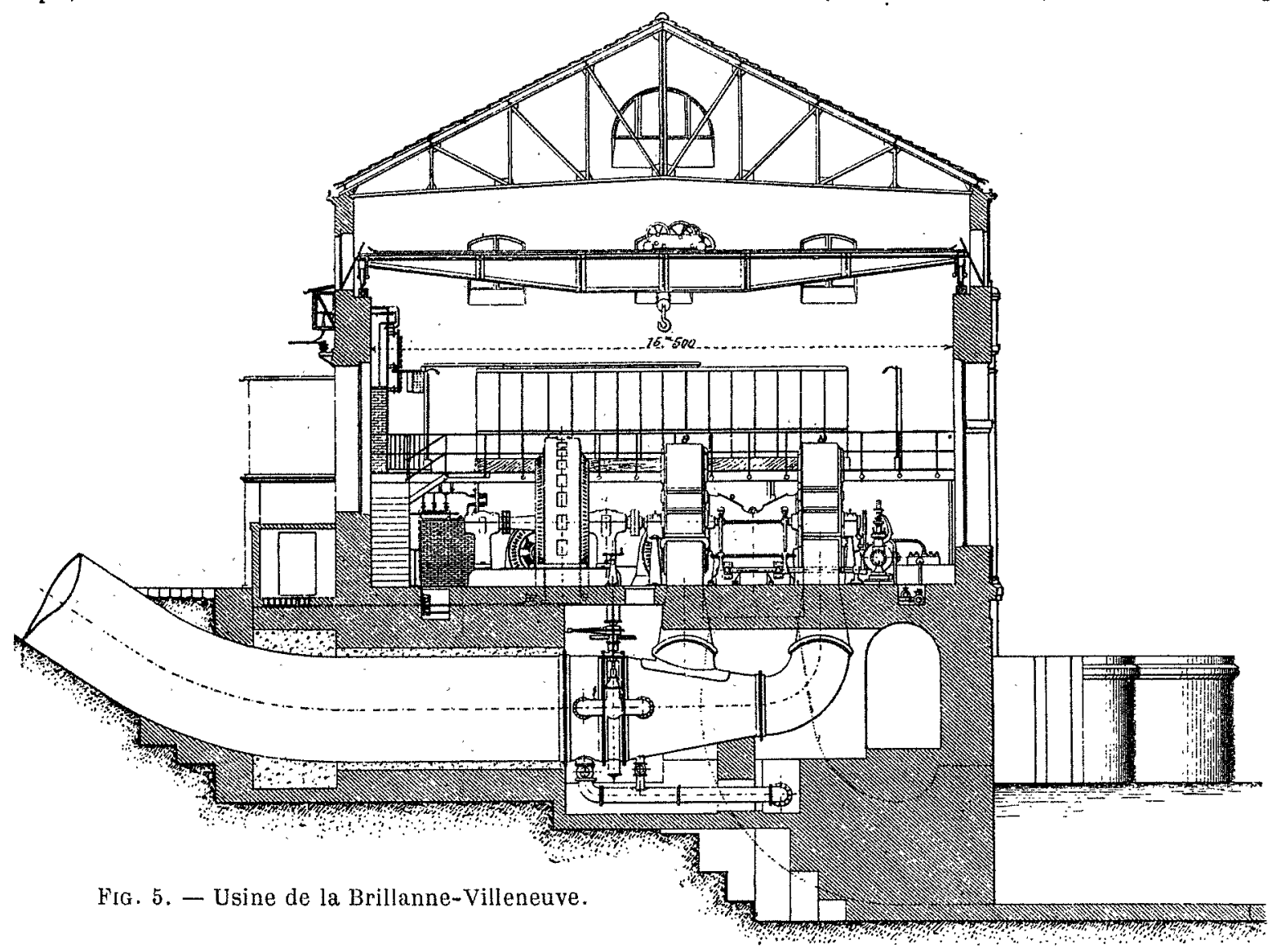
à sa disposition comme appoint.

semble pourra fournir une puissance globable d'environ $60000 \mathrm{HP}$, destinés à alimenter toute la région jusqu'à Marseille. Enfin, trois puissantes usines à vapeur contribueront, dès le début, à l'alimentation de ce vaste réseau.

La première, située à Arles, appartient en propre à l'Ener. gie Electrique et comporte 3 groupes turbo-alternateurs Curtis de $1500 \mathrm{HP}$. La seconde, située à Marseille, appar. tient à la Cie des Tramways, et contient 5 groupes électrogènes de $1500 \mathrm{HP}$, et va être prochainement renforcée par un sixième groupe turbo-alternateur Curtis. La troisième usine, également à Marseille, appartient à la Cie du Gaz, et contient 3 groupes turbo-alternateurs Curtis, de $1500 \mathrm{HP}$, et va être renforcée de deux autres groupes de 1500 et de 5000 HP. Ces deux usines sont à la dispostion de l'Energie Electrique qui, bientôt, aura ainsi près de 25000 chevaux vapeur

Le rése:tu des Bouches-du-Rhône est à la tension primaire de 50000 volts réservées aux grandes artères de transmission. La distribution proprement dite se fait à la tension de 13500 volts, obtenue dans quelques postes de transformation convenablement situés, dont les principaux sont, à l'heure actuelle, à Allauch, près de Marseille, à Arles, et à St-Maximin.

Les lignes primaires sont montées sur poteaux métalliques, de 11 à $12 \mathrm{~m}$. de hauteur au-dessus du sol, et espacés de 70 à $75 \mathrm{~m}$. et même plus. Ces poteaux supportent en général six conducteurs :

3 à 50000 volts, formés de câbles en cuivre de 65 $\mathrm{mm}^{2}$ de section utile, avec âme en jute, disposés, l'un au sommet du poteau, les deux autres sur une traverse métallique et formant avec le premier un triangle équilatéral d'environ $1 \mathrm{~m} 75$ de côté.

3 à 13500 volts, formés de fils de cuivre de 6 à $9 \mathrm{~mm}$ de diamètre, et placés en dessous des précédents.

Les isolateurs à 50000 volts sont en porcelaine, et ne mesurent pas moins de $30 \mathrm{~cm}$. de diamètre maximum sur $30 \mathrm{~cm}$. de hau-

La Société distribue, en outre, l'électricité avec le concour's de la Société lyonnaise d'Applications Electriques, qui assure le service de détail dans les communes de Brignoles Gonfaron, Pignans, Cuers, Carnoules, Besse, Flassans, Sollièr-Toucas, Solliès-Pont, Puget-Ville, Méounes, Belgentier, la Roquebrussane, etc.

\section{RÉSEAU DES BOUCHES-DU-RHONE}

Le réseau des Bouches-du Rhône est alimenté à la fois par l'usine de la Siagne et par l'usine de la Brillanne-Villeneuve, sur la Durance, à laquelle s'adjoindra sous peu une seconde usine sur la Durance, actuellement en construction à Ventavon, près de Gap, et l'usine projetée sur le Verdon au confluent du Jabron, près de Moustiers-Sainte-Marie. L'usine de Ventavon fonctionnera sous une chute de $50 \mathrm{~m}$., et, dès le début, developpera $22000 \mathrm{HP}$. pouvant être beaucoup augmentés ; une fois terminée, elle constituera l'usine hydro-électrique la plus puissante de France. L'em. teur; sans leur suppport ils ne pèsent pas moins de $8 \mathrm{kgs}$ pièce. Ils sont composés de 3 cloches distinctes, assemblées entre elles et au support métallique de l'isolateur par un scellement au ciment Portland. Chaque cloche a été essayée sous une tension de 65 à 70000 volts, et chaque isolateur complet sous celle de 120000 volts.

Usine de La Brillanne-Villeneuve. - Cette usine est alimentée par une dérivation établie sur la rive gauche de la Durance dont le débit est en cet endroit d'environ $60 \mathrm{~m}^{3}$, avec minima, assez rares, de $40 \mathrm{~d} 45 \mathrm{~m}^{3}$. La hauteur de chute utilisée est de 24 mètres.

La prise d'eau se trouve établie à $1600 \mathrm{~m}$. en amont du pont d'Oraison, au pied d'une falaise rocheuse contre laquelle les eaux de la Durance viennent buter naturellement. Grâce à cette disposition des lieux, on s'est contenté de fonder en rivière quatre pertuis de chasse, de $10 \mathrm{~m}$. de largeur, dont les seuils sont arasés à $1 \mathrm{~m} 50$ au-dessous de l'étiage, mais qui peuvent être surélevés au moyen de 
vannes garde-gravier, formées cliacune de 2 éléments de $1 \mathrm{~m}$. de hauteur, qui sont successivement abaissés au moment des crues. A l'étiage, il existe une dénivellation de $1 \mathrm{~m}$. entre les: plans d'eau de la rivière et de l'avantcanal. L'avant-canal à $670 \mathrm{~m}$. de longueur; il est séparé de la rivière par un mur submersible, dans lequel on a ménagé deux prises latérales de secour's, normalement fermées par des barrages à poutrelles. Sur $250 \mathrm{~m}$. de longueur, il a une largeur de $43 \mathrm{~m}$. et forme une vaste chambre à gravier que l'on pourra draguer de temps en temps.

L'entrée du canal proprement dit se fait au moyen de pertuis voûlés qui sont commandés par des vannes métalliques. Ce canal à $6600 \mathrm{~m}$. de longueur; il a une largeur moyenne de $10 \mathrm{~m}$. et un tirant l'eau de $3 \mathrm{~m}$. ; il est entièrement maconné, avec enduit lissé ; sa pente est de 0 m35 par kilomètre, et le débit prévu est de $60 \mathrm{~m}^{3}$ par seconde: sur son parçours il traverse un réservoir de $12000 \mathrm{~m}^{3}$, et aboutit à une chambre d'eau divisée en deux parties par deux grilles de $200 \mathrm{~m}^{2}$ de surface utile; la partie amont étant munie d'un déversoir de $100 \mathrm{~m}$. de longueur, ainsi que de vannes pour évacuer les dépôts. Comme il sera facile d'établir à côté de la chambre d'eau un vaste réservoir de $200000 \mathrm{~m}^{3}$ de capacité, on pourra augmenter sensiblement la puissante instantanée de l'usine au moment du passage des pointes. De la chambre d'eau partent 5 conduites forcées en tòle d'acier, de $2 \mathrm{~m} 70$ de diamètre, etune sixiéme de $1 \mathrm{~m} 20$. Le canal de fuite a un un kilomètre de longueur, $25 \mathrm{~m}^{2}$ de section utile, et une pente de 50 centimètres par kilomètre.

L'usine comporte 5 groupes électrogènes de $3500 \mathrm{HP}$ composés d'un alternateur à induit fixe et inducteur tournant, produisant du courant triphasé à 7500 volts, 25 périodes, et directement accouplés à une turbine double, à axe horizontal et à aspiration du

type Francis, centripète parallèle, tournant à 250 tours par minute et cornposée de deux roues ayant chacune leur arrivée d'eau propre mais ayant une décharge commune (Voir fig. 5). Il y a aussi trois turbines auxiliaires de $350 \mathrm{HP}$ qui commandent: pour la première, un groupe composé par une excitatrice de $200 \mathrm{kws}$ et une dynamo pour services auxiliaires de $50 \mathrm{kws}$; pour la seconde, un alternateur de $250 \mathrm{kws}$ à 13500 volts, destiné au service-de l'éclairage des localités environnntes; la troisième sert de réserve, et commande un groupe et un alternateur semblables aux précédents. Ces turbines sont munies de régulateur's à servo-moteurs à huile, fonctionnant sous une pression de 25 kilogrammes.

Des alternateurs, le courant passe par 15 transformateurs monophasés de $900 \mathrm{kws}$ qui élèvent la tension à 50000 volts; ils sont groupés en triangle du côté basse tension, et en étoile du côté haute tension. Ces transformateurs sont à bain d'huile, avec refroidissement par circulation d'eau. Chaque groupe de transformateur ainsi constitué est relié à deux séries de barres omnibus à 500010 volts d'où partent les lignes se dirigeant soit surArles, soit sur Marseille, d'où partiront également les lignes de raccordement avec les usines de Ventavon et du Verdon,

Les transformateurs, ainsi d'ailleurs que tou t l'appareil- lage, sont logés dans un poste séparé de l'usine. Ce dispositif,analogue à celui des usines d'Arles et de Ventavon, est possible grâce à l'emploi d'interrupteurs commandés à distance. Ces interrupteurs sont analogues à ceux de l'usine de la Siagne et du poste d'Allauch; la rupture est double pour chaque phase. Le réglage de la vitesse des moteurs hydrauliques peut ainsi se faire du tableau mème, de sorle que l'électricien de service possède, concentrés sous sa main, tous les moyens de contrôle et de commande de l'ensemble de l'installation.

Poste d'Allauch. - Le poste d'Allauch, situé près de Marseille, comporte 6 groupes de transformateurs monophasés, de 3000 kw's chacun, abaissant la tension de 50000 à 13500 volts.

La coupure du courant se fait au moyen dinterrupteurs commandés à distance, du type $\mathrm{H}$ de la Compagnie Thomson-Houston. Pour chaque phase, le courant arrive par le fond d'une cuve remplie d'huile, et ressort par le fond d'une autre cuve identique; une fourchetle à deux tiges en cuivre, commandée par une tige verticale isolante, ferme ou ouvre le circuit à l'intérieur des cuves. Le mouvement des trois tiges verticales est effectué par un petit moteur série, fonctionnant sous 110 volts. Les cuves sont útablies en hois spécial, et tapissées à l'intérieur' d'une couche de fihre vulcanisce. Deux rondelles, on fibre digalement, percéns on leur contro pourle passage des liges de contact, sont disposcies pariallèment au plateau circulate formant lo fond de la cuve, et divisent celle-ci en compalliments; tout arc de ruplure tuis'amnrcerait se lrouve coupré, el les remous ou plojeclions d'huile sont supprimés. En outre, chaque cuve repose sur une charpente isolante constituée par des isolateurs en porcelaine, dont la tige de support, en bois spécial, est scellée dans le cadre formant la base de la cellule dans laquelle chaque phase est logée.

Le moteur commande, par l'intermédiaire d'un embrayage magnétique et d'un train d'engrenages, un arbre A relié, par une manivelle $\mathrm{BD}$, à l'un des sommets d'un parallélogramme articulé $\mathrm{DPQK}$, dont le côté $\mathrm{PQ}$ tourne autour d'un axe $O$ fixó au bâti, tandis que le sommel D tourne autour d'un point fixe $\mathrm{O}^{\prime}$, tel que $\mathrm{O}^{\prime} \mathrm{D}=\mathrm{DP}$. Les choses sont disposées de telle sorte que le déplacement vertical de la tige de l'interrupteur correspond à une demirévolution de l'axe $\mathrm{A}$, ét à une rotation de $60^{\circ}$ du point $\mathrm{P}$. On démontre que, dans ces conditions, et pour des longueurs convenables des différents leviers, le point $K$ décrit un arc d'ellipse ayant comme axe la verticale du point $S$, c'est-à-dire qu'en définitive le point $\mathrm{S}$ se meut suivant une ligne droite. Le mouvement progressif de fermelure de l'interrupteur est produit par la rotation de l'arbre A, relié au moteur électrique. La rupture brusque est, au contraire, obtenue par l'action de deux puissants ressorts enroulés autour de l'arbre $Q$, et dont l'une des extrémités est fixée au bras PQ et l'autre à l'arbre lui-mème.

L'axe A tourne toujours dans le même sens, les positions extrêmes de la manivelle $\mathrm{AB}$ étant dans le prolongement 
de la bielle Bo. Les limites de la course du système sont, d'ailleurs, obtenues par deux butées, fixées sur une des roues du train d'engrenages, qui viennent appuyer sur un levier commandé par un électro-aimant de mise en marche. L'arbre A porte, en outre, trois cames dont nous allons voir l'utilité.

Lorsque l'interrupteur est ouvert, la première came de l'arbre A maintient fermé le contact $\mathrm{C}_{1}$. Dans ce cas, la lampe verte $v$ joue le rôle de résistance sur l'électro $\mathrm{E}$ qui ne fonctionne pas. En fermant le commutateur $c$ sur le plot $p$, on excite l'électro qui attire son armature, ce qui ferme le courant du moteur. Tout le système démarre, la deuxième came de l'arbre $\mathrm{A}$ établit le contact $\mathrm{C}_{2}$, tandis que le contact $\mathrm{C}_{4}$ se relève, ce qui désexcite l'électro $\mathrm{E}$ et éteint la lampe verto $v$. On laisse alor's retomber le commutateur $c$. Le mouvement continuant, la deuxième came supprime le contact $\mathrm{C}_{2}$, ce qui coupe le circuit du moteur, la troisième came établit le contact $\mathrm{C}_{3}$ et la lampe rouge $r$ s'allume. En mettant alors le commutateur sur le plot 'p', l'électro s'excite, met le moteur en circuit, ce qui libère tout le système qui se relève brusquement sous l'effort des ressorts; le jeu des contacts est, dans ce cas, tout à fait analogue au précédent.

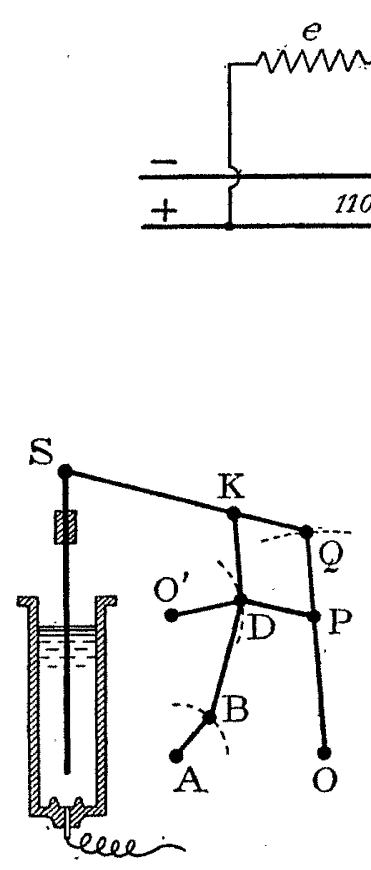

Fig. 7.
L

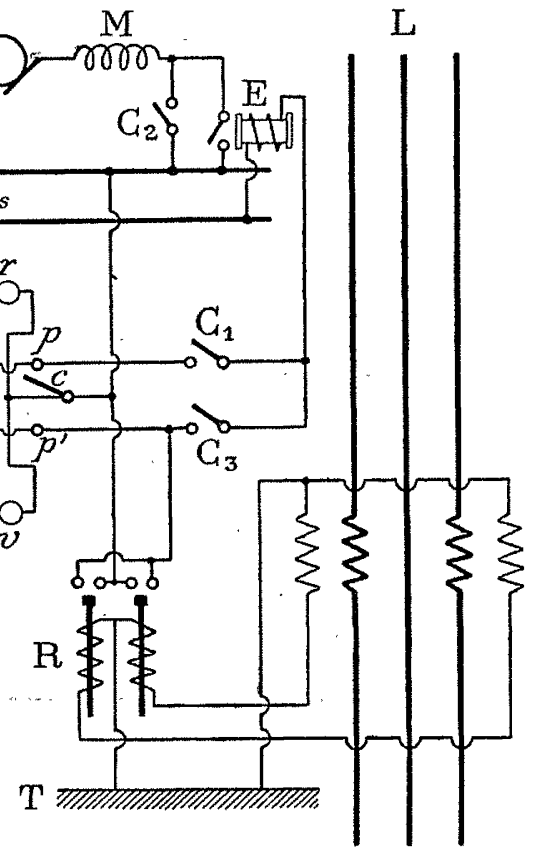

Fig. 8.
Fig. 7 et $8 .-$ Schémas des dispositifs de commande mécanique et électrique de l'interrupteur $\mathrm{H}$.

Enfin, si une surcharge, déterminée à l'avance, vient à se produire, un relai bipolaire à action différée, alimenté par les secondaires de deux transformateurs de courant, agit sur la mise en marche du moteur en excitant l'électro, ce qui provoque le déclanchement du système.

\section{LE SUD ÉLECTRIQUE}

Au moment où l'Energie Electrique installait son réseau des Bouches-du-Rhòne, une contrée d'un intérêt tout spé. cial sollicitait l'attention de la Société, à savoir le Comtat Venaissin et la Camargue, ainsi que les régions viticoles du Gard et de l'Hérault.

A la suite j'un accord avec la Société d'Applications Industrielles, avec la Banque Transatlantique, avec la Société du Sud-Est et avec la Société des Grands Travaux de Marseille, l'Energie Electrique décida de participer à la formation d'une Société ayant pour objet de distribuer de l'énergie électrique dans la région susvisée. Cette Société, appelée le Sud Electrique, a été créée en 1905.
Sous l'active impulsion de son Directeur général, M. Dusaugcy, Je Sud Electrique pousse activement ses travaux; bientôt il alimentera près de deux cents communes, parmi lesquelles nous citerons : Avignon, Nimes, Arles. Tarascon, Carpentras, Aigues-Mortes, ": Cavaillon Saint-Rémy, Orgon, Aramon, Vallabrègues, Châteaurenard, Noves, Eyrargues, Cabannes, Montfrin, Roquemaure, Barbentane, Rognonas, Fontvieille, Paradou, Maussane, Fourques, Marsillargues, Calvisson, Aigues-Vives, Le Grand Gallargues, Milhaud, Vauvert, Aimargues, SaintLaurent, Lansargues, Sorgues, etc. Il fournit, en outre, du courant aux tramways d'Avignon, à la Compagnie d'Eclairage de Montpellier, etc.

Alimenté à l'est par l'Energie Electrique du Littoral Méditerranéen, il le sera, à l'ouest, par la Société des Forces motrices de la Vis, au moyen d'une chute capable de produire 3000 chevaux destinés plus particulièrement à Nimes, Montpellier et ses environs. Cette usine est actuellement en cours d'installation à Madières, commune de Saint-Maurice (Hérault). Le Sud Electrique disposera, en outre, de plusieurs stations à vapeur de secours.

$\mathrm{Au}$ début, le Sud Electrique exploitait provisoirement l'usine de Cavaillon (hydraulique et à vapeur) de 300 chevaux, qui produit du courant triphasé à 50 périodes sous 2700 volts au primaire et 120 volts chez les abonnés. Elle exploite, en outre, deux usines à vapeur, à Tarascon (300 HP) et à Aigues-Mortes (400 HP), ainsi qu'une usine hydraulique et à vapeur, de 500 chevaux, à Marsillargues (Hérault), qui produit du courant alternatif à 50 périodes sous 5000 volts.

Les clients de l'Energie Electrique présentent des régimes de consommation des plus variés. Les uns fonctionnent jour et nuit sans discontinuité, d'autres ne fonctionnent que le jour, les uns avec un régime régulier toute l'année, les autres avec un régime variable avec les saisons, tous ayant les utilisations annuelles les plus diverses. Les puissances absorbées sont, en outre, des plus différentes, et, à côté de la grosse industrie, absorbant jusqu'à des milliers de chevaux, on trouve de petites installations se contentant d'une fraction de cheval.

Pour s'adapter à des besoins aussi variés, la Société a compris qu'il était impossible d'établir une tarification unique, aussi a-t-elle donné à cette tarification une extrême souplesse, susceptible de s'adapter aux variétés infinies de la consommation. Les principales combinaisons offertes par la Société à ses clients sont les suivantes :

10 Tarif simple au compteur, ou tarif mixte, comportant une redevance annuelle déterminée, proportionnelle à la puissance maxima demandée, plus une redevance par kilowatt-heure consommé.

$2^{\circ}$ Forfaits simples basés sur la puissance maxima demandée, ou forfaits avec faculté de dépassement de la puissance maxima dans une proportion déterminée, le supplément étant mesuré par un compteur à dépassement et taxé au kilowatt-heure.

$3^{\circ}$ Double-tarif, au moyen de compteurs change-tarifs enregistrant d'une manière distincte l'énergie consommée à certaines heures de fortes consommations du réseau, et celle consommée le reste de la journée; ou combinaison du double tarif avec les tarifs mixtes ou les forfaits.

Il n'esi pas inutile de faire remarquer, avec $M$. de Marchena, qu'il serait presque impossible à une Administration étatiste (état ou département), exploitant un important réseau de distribution d'énergie, de posséder une telle souplesse dans sa tarification, et de se prêter aussi étroitement aux besoins variés d'une nombreuse clientèle.

\section{H. Bellet.}

